\title{
Adenoid cystic carcinoma of the breast: Review of the literature and report of two cases
}

\author{
SHAOHUA WANG, XIANGJUN JI, YAO WEI, ZEPING YU and NING LI \\ Department of General Surgery, Nanjing Jinling Hospital, Nanjing University, Nanjing, Jiangsu 210002, P.R. China
}

Received April 3, 2012; Accepted July 13, 2012

DOI: $10.3892 / \mathrm{ol} .2012 .818$

\begin{abstract}
In this study, we report two cases of adenoid cystic carcinoma (ACC) of the breast. Two women presented to our hospital with a tender lump in the breast. Mammography and ultrasonography both revealed an ill-defined mass in the breast. We performed modified radical mastectomies on the patients, and pathological assessment following surgery showed ACC of the breast. Both patients received chemotherapy following surgery, have been followed up to date and remain in good health. In this study, we report these two rare cases and review the existing literature regarding ACC of the breast.
\end{abstract}

\section{Introduction}

Adenoid cystic carcinoma (ACC) of the breast is a type of malignant tumor with very low incidence, constituting only $0.1 \%$ of all breast carcinomas (1). In contrast to extramammary ACC, which commonly encroaches the salivary glands, the prognosis of ACC arising in the breast is excellent. This is due to its slower progressive biological course, lower metastases of lymph nodes and uncommon distant metastases $(2,3)$. Generally, it presents with negative estrogen receptors (ER) and progesterone receptors (PR) in immunohistochemical testing. With respect to histologic pathology, its cellular composition comprises ductal epithelioid cells and basaloid myoepithelial cells (4), although there is no commonly accepted origination. Leeming et al showed evidence that ACC originates from the ductal epithelium and myoepithelium (5). Due to its favorable prognosis, accurate clinical diagnosis and differential diagnosis are of significant importance.

The study was approved by the ethics committee of Nanjing University, Nanjing, China. Informed consent was obtained from each patient prior to the study.

Correspondence to: Professor Ning Li, Department of General Surgery, Nanjing Jinling Hospital, Nanjing University, 305 East Zhongshan Road, Nanjing, Jiangsu 210002, P.R. China

E-mail: pht_w@yahoo.com.cn; wanglaifu2@126.com

Key words: breast, adenoid cystic carcinoma

\section{Case reports}

Case 1. A 45-year-old female was admitted to Nanjing Jinling Hospital, China, complaining of a lump in the right breast which had appeared 9 months earlier and had been increasing in tenderness for a week. In the physical examination, the patient's breasts were found to be bilaterally symmetrical, without any skin retraction. Both of the nipples were on the same horizontal line without discharge or retraction. A mass was palpable in the lower-outer quadrant $3 \mathrm{~cm}$ away from the right nipple, with an approximate size of $3 \times 5 \mathrm{~cm}$. The mass was tough in texture, irregular in shape, unclear in boundary and slightly adhesive to surrounding tissues. There were no positive findings in the left breast. The superficial lymph nodes were not palpable in the bilateral axillary and clavicular fossa. The patient had no history of smoking or alcohol consumption and there was no family history of any types of tumor.

Ultrasonography revealed an ill-defined mass in the right breast, and its internal echo was non-homogenous. Mammography revealed a large irregular dense shadow behind the right nipple which was slightly retracted (Fig. 1). The boundary of the shadow was unclear and the glands around were gathered without obvious internal calcification. Additionally, mammography revealed shadows of several hollow swollen lymph nodes in the soft tissue in the anterior of the ipsilateral axilla (Fig. 2). The emission computed tomography (ECT) of whole-body bone imaging showed no signs of tumor metastasis.

The detection of tumor markers showed that serum carbohydrate antigen (CA) 125 was $96.7 \mathrm{IU} / \mathrm{ml}$ and CA $15-3$ was $12.4 \mathrm{IU} / \mathrm{ml}$. Other tumor markers were within normal ranges. Sonographically guided fine-needle aspiration cytology (FNAC) of the mass was performed in another hospital. The pathology of FNAC confirmed a myoepithelial derived tumor, the cells of which were arranged into glandular, cribriform and cord-like patterns. A simple lumpectomy was also performed in the same hospital. The pathology of the specimen identified the tumor as ACC of the breast. Immunohistochemistry of the tumor cells derived from the myoepithelium revealed ER, PR, calponin and smooth muscle actin (SMA) to be positive and P63 to be strongly positive. The patient subsequently underwent a modified radical mastectomy and the pathology which followed showed adenosis of the breast with granulomatous inflammation and a foreign-body giant cell reaction. The margins, basement membrane and nipple were all free 


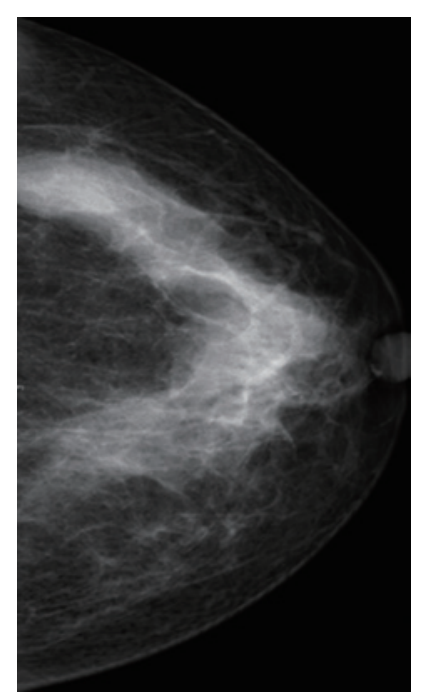

Figure 1. Case 1. Mammography of the right breast showed a large dense shadow behind the right nipple. The boundary of the lump was unclear.

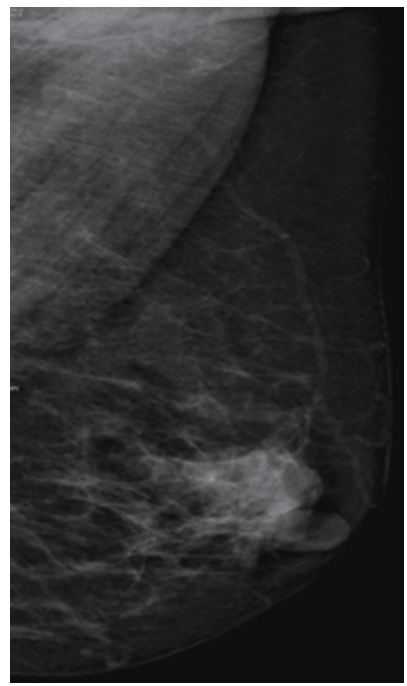

Figure 2. Case 1. There were several swollen lymph nodes in the soft tissue in the anterior of the ipsilateral axilla.

of carcinoma. The lymph nodes were also negative for tumor metastasis.

We performed the modified radical mastectomy according to the pathology results, the patient's economic situation and with the patient's consent. One week after surgery, the patient was given a course of chemotherapy, a combination of cyclophosphamide, pirarubicin and 5-fluorouracil (CEF), by intravenous drip before hospital discharge. The patient subsequently continued her chemotherapy every 3 weeks and is in a good condition to date.

Case 2. A 39-year-old woman was admitted to our hospital due to a tender lump in her left breast which had been present for approximately 3 months. The breast examination revealed a lump under the nipple, approximately $1.5 \mathrm{~cm}$ in diameter. It was tough in texture and immobile; however, there was no erythema, ecchymosis, skin ulceration or dimpling. No axil-
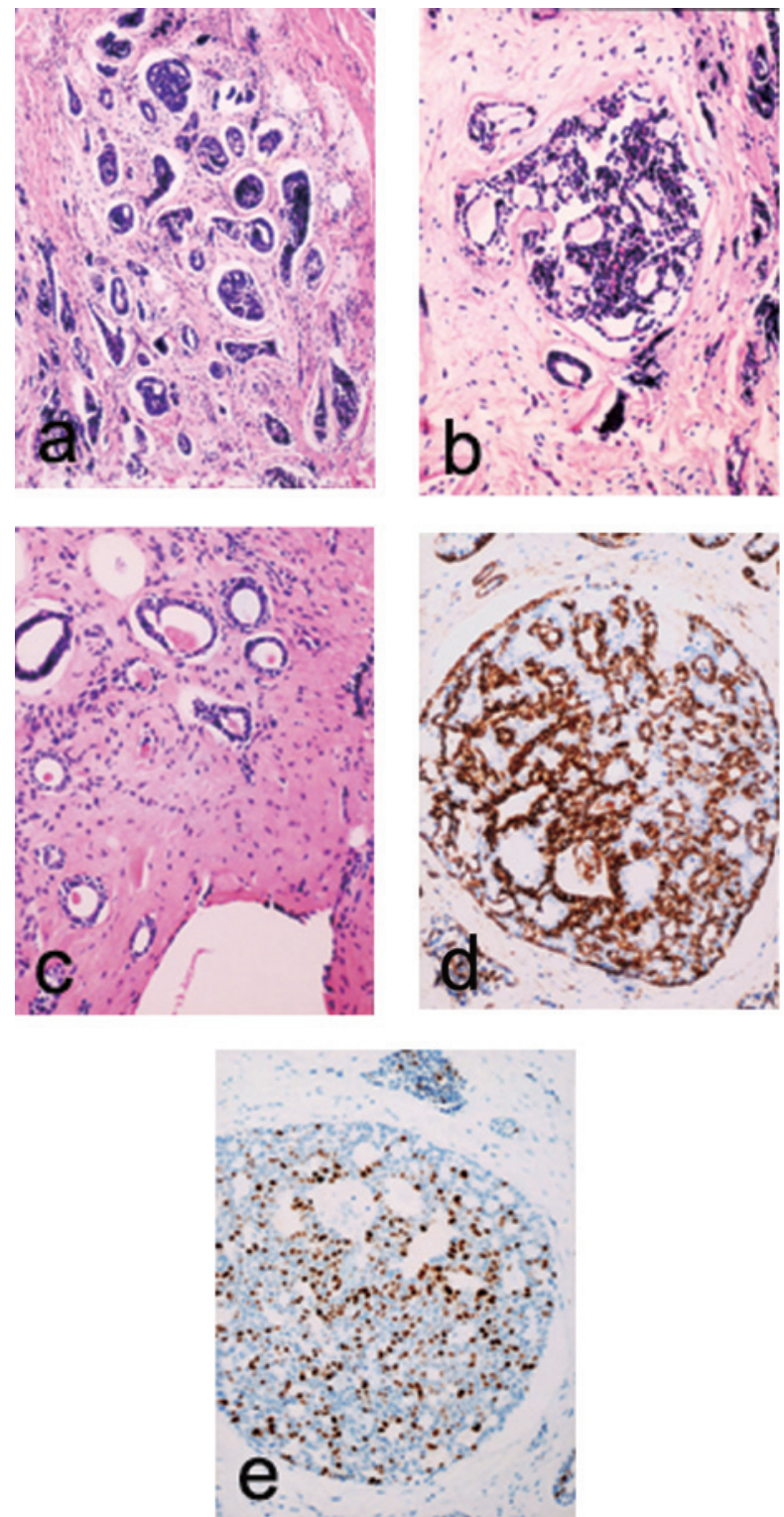

Figure 3. Clinicopathological features of adenoid cystic carcinoma (ACC) of the breast. (a) Myoepithelial cells derived from the tumor arranged into the glandular-cribriform and cord-like patterns (HE, x200); (b) An acidophilic or basopholous mucinous substance inside of cribriform islands (HE, x200); (c) Ducts scattered located around the cribriform structure (HE, x200); (d) SMA, the marker of myoepithelial cells, was positive (SP, x200); (e) Tumor cell proliferation marker Ki67 was highly expressed (SP, x200).

lary lymphadenopathy was detected and there were no positive findings in the laboratory examinations. The family history was negative for breast cancer and the patient was not a smoker or alcohol-consumer.

The patient was given a successful lumpectomy and modified radical mastectomy, and the pathology following surgery showed ACC of the breast. There were no lymph nodes involved. Microscopic examination showed that the tumor cells with different shapes and less cytoplasm formed typical cribriform islands (Fig. 3a), inside of which there was an acidophilic or basopholous mucinous substance (Fig. 3b). Some ducts were scattered and located around the cribriform structure (Fig. 3c). Immunohistochemistry of the tumor cells derived from the glandular epithelium showed that ER, PR and SMA were 
negative and epithelial membrane antigen (EMA) was positive. Tumor cells derived from the myoepithelium showed that SMA was positive and EMA was negative (Fig. 3d). The tumor cell proliferation marker Ki67 was highly expressed (Fig. 3e). The patient received two courses of chemotherapy (CEF), and remained in a good condition throughout the 28-month follow-up period.

\section{Discussion}

ACC of the breast is a rare and slowly progressive carcinoma accounting for $0.1 \%$ of all breast carcinomas. It is of note due to its favorable prognosis and distinctive histological appearance, since lymph node involvement and distant metastases are very uncommon. Predominantly, the neoplasm affects women at a mean age of 50-65 years (1). According to existing literature, it mainly occurs unilaterally without predilection for laterality (1).

The most common clinical manifestation of ACC of the breast is a tender mass, commonly located in the area of the areola, as was demonstrated in our patients. The nipple is seldom involved and nipple discharge is uncommon. Spontaneous pain in a patient may be attributable to the perineural invasion of the tumor; however, this symptom is uncommon. McClenathan et al retrospectively analyzed 22 cases and discovered that 10 patients primarily complained of pain (6). Several of them felt extreme pain while others complained of local pain at least one year before an abnormality was found using medical imaging. Notably, perineural invasion of the tumor was not evident among the patients experiencing spontaneous pain. In our second case, the patient complained of tenderness of the lump, which had been found in the right breast several months previously.

A review of the existing literature revealed that the imaging features of ACC of the breast were not as typical and symptomatic as the clinical manifestation. X-ray radiological imaging may present ACC as a benign tumor, with a smooth and clear boundary and regular density, or with a lobulated density and uneven density shadow. Santamaría et al, combining imaging appearances with pathological characteristics, discovered that the majority of carcinomas were located in the upper quadrant of the breast or in the area of the areola (7). In mammography, ACC may present as a circumscribed lobulated nodule. When the boundaries of the nodular shadow were unclear or partially unclear, microscopic invasion was usually found (7). Inside the tumor lesion there may be calcifications, which are generally observed in histology but infrequently detected in mammography. Saqi et al noted that it was significant to make full use of FNAC for the early diagnosis of ACC of the breast (8).

Lymph node metastases as well as distant metastases of ACC in the breast are rare. Sumpio et al reviewed 120 cases from the literature and showed lymph node metastases in only 4 cases, and distant metastases in only 8 cases, the majority of which occurred in the lung (9). In another series of 182 cases, Arpino et al noted fewer than 10 cases with lymph node metastases and only 14 cases with distant metastases in the 10 years following surgery (10). However, distant metastases may occur, most frequently in the lung, without sentinel lymph node involvement. In the present case reports, all the lymph nodes sent for pathological biopsy were negative for tumor cells.
With regard to pathology, ACC of the breast is mainly composed of two types of cells; ductal epithelioid cells and basaloid myoepithelial cells, which form circumscribed clusters of cells arranged in solid, cribriform, tubular and trabecular arrangements, although one pattern may predominate (4). According to its various growth patterns, ACC of the breast may be classified as cribriform, tubular or solid types. The cribriform type is the most symptomatic and most frequently presented, while the solid type occurs comparatively seldom (11). The differential diagnosis of the carcinoma includes cribriform ductal carcinoma in situ, invasive cribriform carcinoma, invasive ductal carcinoma and benign tumors such as collagenous spherulosis. The negative expression of ER and PR helps to distinguish ACC from cribriform ductal carcinoma in situ, which lacks the basement membrane-like material found in the lumens of ACC. Invasive cribriform carcinomas and ductal carcinomas in situ also present ER and PR; however, the invasive cribriform carcinoma lacks myoepithelial cells related to its invasive characteristics. The collagenous spherulosis has acidophilic spherules rich in collagen, which have positive PAS (Periodic Acid-Schiff) staining and accompany usual type epithelial hyperplasia. ACC of the solid type is similar to ordinary ductal carcinoma. Acs et al reported 17 cases of ACC coexisting with microglandular adenosis (MGA) and suggested that ACC may develop in the background of and in continuity with MGA (12).

As the prognosis of ACC is related to the histological grade, Ro et al (13) suggested that ACC of the breast may be classified into three grades according to the proportion of solid growth elements of the tumor. Tumors of grade I are made up of glandular and ductal structures without any solid elements. Tumors of grade II include less than $30 \%$ solid elements and grade III tumors include more than $30 \%$. This grading was found to be of great significance in treatment and prognosis. As the percentage of solid elements increases, so does the risk of tumor development and postoperative recurrence $(3,7)$

Arpino et al (10) reviewed the molecular markers, treatment and clinical outcomes of 28 cases of ACC of the breast. Having analyzed the DNA of the tumors, they found that $92 \%$ of the patients were DNA diploid, whereas the frequency of DNA aneuploidy in invasive breast carcinomas was approximately 60-65\%. AAC of the breast also showed lower proliferative activity with PR and ER usually negative. Leeming et al (5) reviewed 140 cases and found only one case positive in ER. However, in a different group, which consisted of 14 cases, there were 4 positive in ER and in a separate group of 13, there were 3 positive in PR (6). Similarly, Kleer et al (14) analyzed 19 cases and found 5 positive in ER. In this case, both ER and PR were positive in the tumor cells. Based on the above, ACC of the breast may not be completely distinguished from other invasive breast carcinomas according to ER and PR expression alone.

The optimal treatment of ACC of the breast has not yet been determined due to its low incidence. The reviewed literature shows several surgical treatment modalities ranging from a simple lumpectomy without radiotherapy to a radical mastectomy. The radical mastectomy is not advised due to the high risk of physical and psychological damage to patients and the low incidence of nodal metastasis of the tumor. McClenathan et al (6) suggested that even limited lymph node 
dissection was unwarranted. Instead, the simple mastectomy has widely been used and is preferred by many surgeons. The simple lumpectomy has the advantages of lower operative damage, less postoperative discomfort and faster recovery; however, it is associated with unacceptably high rates of local recurrence. As a result, it is necessary to combine the lumpectomy with adjuvant systemic chemotherapy or local radiotherapy with discretion. According to the pathological grading suggested by Ro et al (13), the proposed treatment is simple lumpectomy for grade I tumors, simple mastectomy for grade II tumors and mastectomy with axillary clearance for grade III tumors. With respect to adjuvant chemotherapy, hormonal manipulation and bio-immunotherapy treatments of ACC of the breast, there are still few studies to date. To find the optimum treatment, prospective clinical trials need to compare the different treatments, which may prove difficult to conduct in such a rare neoplasm.

There are several studies which have investigated the postoperative recurrence rates of ACC of the breast. Literature overviews revealed higher rates of local recurrence following simple lumpectomy. Santamaría et al (7) reviewed 182 cases following surgery and found 14 cases of local recurrence, of which 11 recurred after simple lumpectomy, one after simple mastectomy and 2 after radical or modifed mastectomy. Sumpio et al (9) reported 6 cases of local recurrence following simple lumpectomy, out of a total of 8 cases. Leeming et al (5) noted that 9 out of 24 patients suffered from local recurrence following simple lumpectomy, a recurrence rate of $37 \%$. Youk et al (15) described a case of local recurrence following successful treatment by lumpectomy and systemic chemotherapy and radiotherapy.

ACC of the breast is a type of rare carcinoma with relatively typical clinical presentation. The diagnosis may be made by the combination of imaging and pathology; however, there is still no consensus on the standard treatment. Despite the favorable prognosis, there are reports of local recurrences and distant metastases following treatment. As a result, it is necessary for the patients to be closely followed-up and periodically examined following treatment.

\section{References}

1. Law YM, Quek ST, Tan PH and Wong SL: Adenoid cystic carcinoma of the breast. Singapore Med J 50: e8-e11, 2009.

2. Cavanzo FJ and Taylor HB: Adenoid cystic carcinoma of the breast: an analysis of 21 cases. Cancer 24: 740-745, 1969.

3. Peters GN and Wolff M: Adenoid cystic carcinoma of the breast. Report of 11 new cases: Review of the literature and discussion of biological behavior. Cancer 52: 680-686, 1983.

4. Azoulay S, Laé M, Fréneaux P, Merle S, et al: KIT is highly expressed in adenoid cystic carcinoma of the breast, a basal-like carcinoma associated with a favorable outcome. Mod Pathol 18: 1623-1631, 2005

5. Leeming R, Jenkins M and Mendelson G: Adenoid cystic carcinoma of the breast. Arch Surg 127: 233-235, 1992.

6. McClenathan JH and de la Roza G: Adenoid cystic breast cancer. Am J Surg 183: 646-649, 2002.

7. Santamaría G, Velasco M, Zanón G, Farrús B, et al: Adenoid cystic carcinoma of the breast: mammographic appearance and pathologic correlation. Am J Roentgenol 171: 1679-1683, 1998.

8. Saqi A, Mercado CL and Hamele-Bena D: Adenoid cystic carcinoma of the breast diagnosed by fine-needle aspiration. Diagn Cytopathol 30: 271-274, 2004.

9. Sumpio BE, Jennings TA, Merino MJ and Sullivan PD: Adenoid cystic carcinoma of the breast. Data from the Connecticut Tumor Registry and a review of the literature. Ann Surg 205: 295-301, 1987.

10. Arpino G, Clark GM, Mohsin S, Bardou VJ, et al: Adenoid cystic carcinoma of the breast: molecular markers, treatment and clinical outcome. Cancer 94: 2119-2127, 2002.

11. Yang WT, Zhang TQ and Shen MC: Adenoid cystic carcinoma of the breast: report of four cases and review of the literature. J Clin Exp Pathol 21: 10-13, 2005.

12. Acs G, Simpson JF, Bleiweiss IJ, Hugh J, et al: Microglandular adenosis with transition into adenoid cystic carcinoma of the breast. Am J Surg Pathol 27: 1052-1060, 2003.

13. Ro JY, Silva EG and Gallager HS: Adenoid cystic carcinoma of the breast. Hum Pathol 18: 1276-1281, 1987.

14. Kleer CG and Oberman HA: Adenoid cystic carcinoma of the breast: value of histologic grading and proliferative activity. Am J Surg Pathol 22: 569-575, 1998.

15. Youk JH, Kim MJ, Kim EK, Lee JY, et al: Recurrence of adenoid cystic carcinoma in the breast after lumpectomy and adjuvant therapy. J Ultrasound Med 25: 921-924, 2006. 